\title{
Kernos
}

Revue internationale et pluridisciplinaire de religion grecque antique

2 | 1989

Varia

\section{Rhésos ou la quête de l'immortalité}

\section{Paul Wathelet}

Édition électronique

DOI : $10.4000 /$ kernos.250

ISSN : 2034-7871

\section{Éditeur}

Centre international d'étude de la religion grecque antique

\section{Édition imprimée}

Date de publication : 1 janvier 1989

Pagination : 213-231

ISSN : 0776-3824

\section{Référence électronique}

Paul Wathelet, "Rhésos ou la quête de l'immortalité », Kernos [En ligne], 2 | 1989, mis en ligne le 02 mars 2011, consulté le 20 avril 2019. URL : http://journals.openedition.org/kernos/250; DOI :

$10.4000 /$ kernos.250 
Kernos, 2 (1989), p. 213-231.

\section{RHÉSOS OU LA QUÊTE DE L'TMMORTALITÉ}

Depuis l'Antiquité, le dixième chant de l'Iliade suscite la méfiance et son authenticité est mise en doute ${ }^{1}$. Il ne s'agit pas de prendre ici le contre-pied de toute la tradition et tenter de démontrer à toute force que la Dolonie est incontestablement ce qu'Homère a fait de mieux. Je reconnais bien volontiers, après beaucoup d'autres, qu'il s'agit d'un hors-d'œuvre, dont le ton général est différent de celui du reste de l'Iliade. Je vais néanmoins prendre quelque peu la défense du passage et m'efforcer de montrer que la Dolonie répond au moins à une certaine logique interne qui, me semble-t-il, n'a guère été soulignée jusqu'à présent.

Que le morceau s'intègre mal dans l'Iliade que nous possédons est un fait d'évidence ${ }^{2}$. Tout le chant IX se passe de nuit, c'est le célèbre épisode des

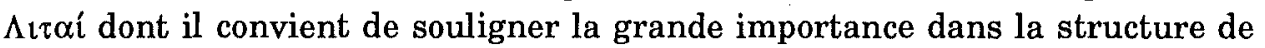
l'œuvre $^{3}$. Angoissés par la situation militaire (les Troyens menacent les vaisseaux de près), les chefs achéens ont tenu conseil; ils ont envoyé trois ambassadeurs auprès d'Achille pour apaiser sa colère; les ambassadeurs ont échoué. À la fin du chant IX, tout le monde va se coucher. Au début du chant X, c'est toujours la nuit et les guerriers souffrent d'une insomnie persistante.

Les chefs achéens se réunissent de nouveau et ils décident d'envoyer deux d'entre eux en éclaireurs afin d'espionner les Troyens et de voir leur

1 Cf. le Schol. T au début du chant qui accuse Pisistrate d'avoir introduit le passage dans l'Iliade.

2 L'opinion selon laquelle la Dolonie est un morceau à part qui s'intègre mal au plan de l'Iliade est quasi universelle chez les homérologues (C. WHITMAN, Homer and the Heroic Tradition, Cambridge [Mass.], Harvard Univ. Press, 1958, p. 283). Les partisans du passage considéré comme une interpolation tardive constituent la majorité (cf. E.R. DoDDs, Homer, in Fifty Years of Classical Scholarship, Oxford, Blackwell, 1954, p. 1-17 et p. 31-35, repris in G.S. KIRK, The Language and Background of Homer, Cambridge, Heffer, p. 4 et n. 12). A. SHEWAN, The Lay of Dolon, Londres, Macmillan, 1911, a défendu le passage contre ceux qui l'accusaient d'être récent. Bien que ses arguments soient souvent bons, il n'a guère été suivi. Chez certains spécialistes, cette exclusion s'ajoute à celle d'autres passages. On se demandera, avec A. Schnapp-Gourbeillon (Lions, héros, masques. Les représentations de l'animal chez Homère, Paris, Maspero, 1981, p. 105, n. 11), ce qui resterait de l'Iliade si on en enlevait tous les passages suspectés !

3 D. AUBRIOT, Remarques sur le personnage de Phénix au chant IX de l'Tliade, in $B A G B$ (1984), p. 339-363; Remarques sur le chant IX de l'Tliade, in BAGB (1985), p. 257-280. 
dispositif militaire. Diomède sera accompagné par Ulysse. On prête des armes aux deux héros qui en sont dépourvus. Diomède et Ulysse, encouragés par un présage favorable d'Athéna, s'enfoncent dans la nuit.

Mus sans doute par une inquiétude du même genre, les Troyens tiennent conseil et décident, eux aussi, d'envoyer un espion reconnaître les lignes achéennes. Un inconnu se présente, un certain Dolon, fils d'Eumédès. Pour prix de son offre, Dolon réclame d'Hector un cadeau invraisemblable : le char et les chevaux d'Achille. Hector appuie sa promesse d'un serment dont l'aède prend soin de nous dire qu'il n'aura pas lieu d'être observé.

Dolon, qui a revêtu la dépouille d'un loup gris et s'est coiffé d'une peau de martre, s'engage dans la nuit.

Les deux Achéens et le Troyen vont se rencontrer ou plutôt, grâce à une ruse d'Ulysse, Dolon croise Diomède et Ulysse sans les voir et ceux-ci lui coupent la retraite : Dolon est fait prisonnier. Vert de peur, il décrit, sans trop se faire prier, le dispositif militaire des Troyens et finit par annoncer que le bord extrême du bivouac troyen est occupé par les Thraces, arrivés le soir même sous la conduite de leur roi, Rhésos.

Ayant tiré de Dolon tout ce qu'ils pouvaient en apprendre, Diomède le tue et Ulysse dispose ses dépouilles sur les branches d'un tamaris. Puis les deux Achéens gagnent le camp des Thraces, qui sont plongés dans un profond sommeil. Chacun est couché près de son char et de ses chevaux. Diomède en tue douze, tandis qu'Ulysse écarte les corps afin que le passage soit libre. Le treizième Thrace qu'ils tuent est Rhésos lui-même, aussi endormi que ses compatriotes et qui est justement en proie à un cauchemar, envoyé par Athéna. Il rêve que Diomède est en train de le tuer. Rhésos mort, les Achéens s'emparent de ses chevaux; Diomède est sur le point de prendre aussi le char du roi, mais Athéna intervient pour l'en dissuader. Apollon veille et il vient de réveiller un cousin de Rhésos, Hippokoon qui donne l'alarme. Diomède et Ulysse se sauvent, chevauchant les coursiers de Rhésos. Au moment où ils passent le fossé du camp achéen, ils sont accueillis par Nestor, qui admire leur monture. Les deux héros prennent ensuite un double bain, dans la mer, puis dans une baignoire.

Tel est brièvement résumé le récit de la Dolonie. Il a fait l'objet de critiques nombreuses, dont un certain nombre au moins ne sont pas dépourvues de fondement. Au début, il est dit que Diomède et Ulysse vont aller espionner les Troyens ${ }^{4}$, alors que, dans la suite, ils s'avancent dans le camp ennemi et tuent Rhésos. On ne voit pas le lien entre la mort de Dolon et

4 Dans l'invocation qu'il adresse à Athéna, Diomède parle de $\mu$ é $\rho \varepsilon \rho \alpha(X, 299)$ qui n'étaient pas prévus au départ. 
celle de Rhésos 5 . Diomède et Ulysse auraient pu être prévenus autrement du dispositif d'installation des Thraces que par l'épisode de Dolon, dont on ne voit pas ce qu'il apporte. De surcroît, tout le passage est surchargé de détails difficiles à expliquer : pourquoi les deux guerriers achéens revêtent-ils des armements qui ne sont pas les leurs, pourquoi Dolon s'affuble-t-il de la peau d'un loup gris et de celle d'une martre, etc. ?

Comme souvent, pour comprendre le texte, il convient de le lire et de le relire, en en considérant tous les détails. Reprenons le morceau qui, jusqu'à présent, a été résumé. Tout le début du chant est marqué par l'angoisse qui règne dans le camp achéen. Désespéré, Agamemnon adresse des reproches à Zeus (82). Dans l'Iliade, l'Atride n'est certes pas un personnage sympathique, mais il est remarquable que le roi de Mycènes, chef de l'expédition, et qui, par le fait même, devrait être proche de Zeus, se trouve en mauvais termes avec le Cronide ${ }^{6}$. Agamemnon va réveiller Nestor ${ }^{7}$. Celui-ci est le plus âgé des chefs achéens; protégé de Poséidon, Nestor a de fortes connotations chthoniennes. Il règne sur la «Pylos des sables», dont le nom même évoque l'entrée de l'Autre Monde. Vieillard bavard, il ne cesse de dispenser ses conseils. Déjà dans les Litai, Nestor a pris l'initiative de diriger les opérations alors qu'Agamemnon, découragé, a abandonné ses responsabilités de roi suprême. En quelque sorte, au chant X, comme au chant IX, on a le sentiment d'une vacance du pouvoir dans une période décisive et particulièrement délicate, où les Achéens, comme le dit le texte, sont "sur le fil du rasoir» $(X, 173)$.

Les chefs achéens vont tenir conseil aux avant-postes, à l'endroit même où les Troyens se déchaînaient quelques heures auparavant ${ }^{8}$ et c'est Nestor

5 B. FENIK, Iliad $X$ and the Rhesos. The Myth, Bruxelles, Latomus, 1964, s'efforce de dissocier les deux parties de la Dolonie et de montrer que l'épisode de Dolon n'est que le résultat d'une reprise maladroite d'un vieux récit, dont la tragédie attribuée à Euripide donnerait une version plus ancienne. Le personnage de Dolon semble gêner B. Fenik, comme d'ailleurs l'auteur de la tragédie.

6 Sur Agamemnon, cf. P. WATHELET, Dictionnaire des Troyens de l'Iliade, à paraître,

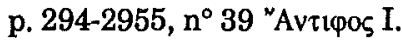

7 Sur l'étymologie du nom de Nestor, cf. C.J. RUIJGH, Études sur la grammaire et le vocabulaire du grec mycénien, Amsterdam, Hakkert, 1967, p. 369-370, § 335. - Sur le lien entre Nestor et Poséidon, cf. $I l$., XIII, 554-563.

8 Ici encore, les détails donnés par le récit sont curieux : pourquoi les chefs achéens tiennent-ils conseil en dehors de leur camp, au milieu du champ de bataille en un endroit qui n'est pas encombré de cadavres? Après avoir souligné l'angoisse qui pèse sur le camp, l'aède souligne que les chefs achéens passent le fossé. Ils prennent donc au moins le risque d'une attaque surprise des Troyens. On pourrait penser qu'ils veulent s'isoler de leurs troupes et qu'ils sont à ce point pressés par les événements 
qui va suggérer l'envoi d'un éclaireur; il propose que la récompense soit une brebis noire. La couleur de la brebis montre que l'on est dans le domaine souterrain.

Les Achéens décident donc de désigner un espion. Le premier choisi est Diomède ${ }^{9}$. Celui-ci se fera accompagner par Ulysse «parce que, lui aussi, est cher à Athéna» 10 . Nous sommes donc en présence de deux héros patronnés par Athéna. Le fait est incontestable pour chacun des deux dans toute l'œuvre homérique. On se souvient qu'au chant $\mathrm{V}$ Diomède a remporté d'éclatants succès, grâce à l'appui continu de sa déesse tutélaire. Celle-ci a été jusqu'à prendre la place de son cocher, Sthénélos, pour lui donner la victoire, bien que la position de cocher soit normalement subalterne. Quant à Ulysse, il suffit de jeter un coup d'œil sur l'Odyssée pour voir combien il est en permanence favorisé par la même déesse.

Diomède, dont le nom semble signifier la "pensée de Zeus", est un des rares noms théophores que connaît l'Iliade ${ }^{11}$. L'anthroponyme désigne deux

qu'ils n'ont d'autre endroit pour se tenir que le champ de bataille. Toutefois, ailleurs dans l'Iliade, les chefs se réunissent entre eux (c'est le cas au chant I et au début du chant II) sans sortir du camp. S'agit-il d'occuper symboliquement un endroit où Hector, pressé par la nuit, a dû arrêter son avance ? Y aurait-il là quelque geste rituel, peut-être apotropaïque, qu'une version plus ancienne du récit aurait précisé, mais que l'auteur de l'Iliade aurait gommée, ainsi qu'il en a l'habitude ? Ceci donnerait à penser que la Dolonie a été adaptée par Homère. Etant donné qu'on sera amené à conclure, de l'ensemble de l'expédition de Diomède et d'Ulysse, qu'il s'agit d'une descente aux Enfers, on peut se demander si les chefs achéens ne sortent pas du camp, comme, au XXIVe chant, les Troyens accompagnent Priam qui se rend chez Achille (P. WATHELET, Priam aux Enfers, in LEC, 56 [1988], p. 320-335) ou comme on sortait des murs de la cité pour suivre un défunt que l'on conduisait, hors les murs, à son tombeau.

9 Agamemnon répond d'autant plus favorablement à la demande de Diomède qu'il craint pour Ménélas $(X, 240)$ dont le personnage est spécialement critiqué depuis le début du chant (114-123). Dans le climat d'angoisse qui étreint les Achéens et alors qu'un événement capital se prépare, la responsabilité de Ménélas dans l'expédition est soulignée sans ménagement.

10 La tradition posthomérique associera les deux héros au moins dans une autre aventure, le rapt de Palladion. Parfois, dans les deux entreprises, un élément de comique sera glissé qui les opposera, Ulysse tentant de duper Diomède. Dans la légende de Philoctète, le rôle dévolu à Diomède (Petite Iliade, Résumé de Proklos, 212-213, A. Severyns) est ultérieurement repris par Ulysse (SoPHOcLE, Philoctete). Diomède est présenté comme plus robuste et plus noble, mais moins rusé qu'Ulysse, avec peut-être un côté plus complexe et plus inquiétant que le fils de Laërte, lequel est plus proche d'Athéna. Le rapprochement des deux héros sera fait au détriment de Diomède.

11 H. VON KAMPTZ, Homerische Personennamen. Sprachwissenschaftliche und historische Klassifikation, Göttingen, Vandenhoeck \& Ruprecht, 1958, p. 39-40, \& 12; 
héros, le fils de Tydée, le nôtre, et un autre Diomède, fils d'Arès et roi des Bistones, c'est-à-dire d'un peuple thrace. Alors que le fils de Tydée est un héros enviable et glorieux, son homonyme thrace est un personnage peu recommandable. C'est lui qui nourrissait des cavales anthropophages dont Héraklès devait purger l'univers, donnant même, dans certaines versions, le roi à manger à ses propres cavales ${ }^{12}$.

Ulysse est un très vieux héros préhellénique comme son nom d'indique :

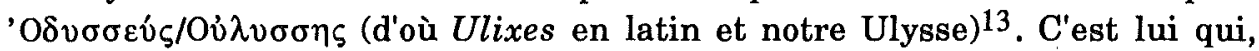
comme les héros des imrama celtiques ou, au moyen âge, saint Brendan, se rend dans l'Autre Monde. Nous ignorons ce qu'il allait y chercher à l'origine. Dans l'Odyssée homérique, l'épisode a été rattaché à la guerre de

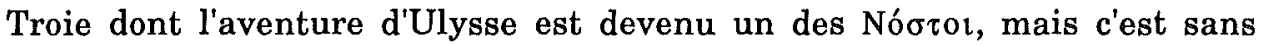
doute là une conception relativement tardive, dont la célèbre Nekyia constitue un reste ${ }^{14}$.

Par une singulière coïncidence, les Troyens tiennent une assemblée et ils décident également d'espionner leurs ennemis : un candidat se présente, il s'agit de Dolon. À l'inverse de Diomède et d'Ulysse chez les Achéens, Dolon est un personnage inconnu que l'aède éprouve le besoin de présenter aux vers 314-317:

Il y avait chez les Troyens un certain Dolon, fils d'Eumédès

Héraut divin, riche en or, riche en bronze

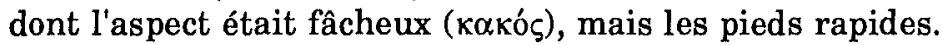

Il était seul fils, auprès de cinq sœurs.

Ne voyons pas, dans cette accumulation de précisions, un ensemble de touches pittoresques qui donneraient éventuellement un genre de couleur locale au tableau. Tous les détails ont une valeur et ils convergent. Dolon

p. $89, \S 31$ a $2 ;$ p. $189, \S 66$. - Cf. P. Wathelet, Dict. des Troyens, p. 135-138, $\mathrm{n}^{\circ} 2$ "A $\beta \alpha \varsigma$.

12 Il est probable que, comme l'a suggéré depuis longtemps E. BETHE, art. Diomedes 1 in $R E, \mathrm{~V}$ (1903), c. 815-826, les deux Diomède ne sont, à l'origine, qu'un seul et même héros (malgré le scepticisme de Ø. ANDERSEN, Die Diomedesgestalt in der Ilias, Oslo, Universitesforleget, 1978), dont le méchant côté a été attribué au roi des Bistones, c'est-à-dire à un étranger, tandis que l'aspect favorable devenait l'apanage du héros grec. Le premier est lié à Arès, divinité que les Grecs écartent, tandis que le second est sous la protection d'Athéna.

13 H. VON KAMPTZ, p. 335-357, \& 83.

14 Il est sans doute dangereux d'appliquer notre logique à un texte aussi ancien, mais on peut s'étonner qu'Ulysse se donne la peine extrême d'aller jusqu'à l'entrée de l'Autre Monde uniquement pour consulter le devin Tirésias sur son itinéraire de retour. On a le sentiment que la Nekyia est une sorte de passage obligé imposé par la tradition. 


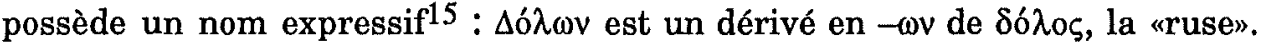
Dolon est donc le "rusé». Son nom est grec. Il est fils d'Eumédès, autre nom hellénique; Eumédès est le «bienveillant», de $\varepsilon \dot{v}$ - bon, bien - et de $\mu \dot{n}^{\prime} \delta \alpha_{\alpha \iota}$ qui marque une activité de l'esprit ${ }^{16}$. Eumédès était héraut divin; nous ignorons à quoi correspondait cette fonction, si elle faisait allusion à quelque épisode de la geste qui ne nous serait pas connu. Sans aucune autre précision, la mention de «héraut divin" évoque une divinité, Hermès, héraut des dieux. Dolon est riche, il possède beaucoup d'or et beaucoup de bronze, en quelque sorte une richesse métallique. Dolon ne se range pas dans l'aristocratie

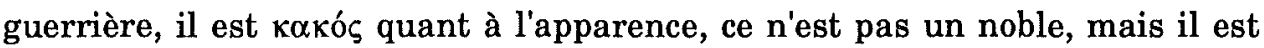
rapide à la course. Un dernier détail souligne qu'il n'est pas très viril : il est seul fils à côté de cinq sœurs. On peut imaginer que, dans un pareil entourage, notre héros n'a guère été formé aux entreprises guerrières. La situation exactement opposée est illustrée par Andromaque. La femme d'Hector rappelle en effet, au chant VI (421-422), qu'elle avait sept frères ${ }^{17}$. $\mathrm{Au}$ contact de tous ses frères, Andromaque a pu se forger le caractère; et de fait, elle possède un nom qui signifie "celle qui combat l'homme» et l'analyse de son rôle dans la tradition grecque montre qu'elle se conduit comme une Amazone ${ }^{18}$. Dolon risque done de manquer de courage. La mention des cinq sœurs apporte une autre indication qui conforte ce que l'aède vient de dire. À l'époque des héros d'Homère, l'usage est, pour un fiancé, d'acheter sa future femme, qu'il paie parfois très cher, offrant pour prix de sa main des bœufs nombreux, d'où les noms d'héroïnes comme Périboia, "celle qui a coûté beaucoup de bœufs» ${ }^{19}$. Les cinq sœurs de Dolon pouvaient sans doute prétendre faire de riches mariages et Eumédès aurait trouvé dans les cadeaux apportés par les futurs maris une belle occasion de s'enrichir.

Tous les caractères prêtés à Dolon renvoient en fait à une divinité déterminée, Hermès ${ }^{20}$. Le dieu est rusé, il possède l'adresse des commerçants qui tentent de vendre leur marchandise, il est bienveillant pour les mêmes

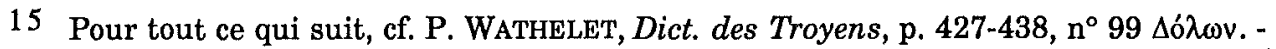
Sur le nom, H. vON KAMPTZ, p. 26, § 10 a $1 ;$ p. 133, § 43 b $3 ;$ p. 239-40, § 68 c 2 .

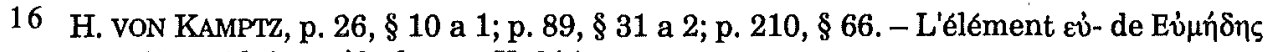
peut être réduit en nंvे-de $v$ en $X, 314$.

17 A. Moreau, Etymologie et mythe originel : Adraste, Andromaque, Déjanire, in Sens et pouvoirs de la nomination in les cultures hellénique et romaine. Actes du colloque de Montpellier, 23-24 mai 1987, Montpellier, 1988, p. 114-116.

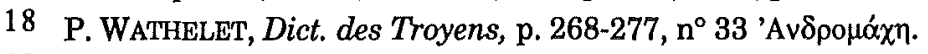

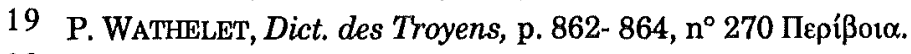

20 W. BURKERT, Greek Religion. Archaic and Classical, Oxford, Blackwell, p. 156-159. 
raisons (il faut aguicher les chalands). Hermès est aussi un dieu chthonien associé aux richesses de la terre et notamment aux richesses métalliques. Parfois, à Samothrace, on le confondra avec les Cabires, groupe de divinités mal connues, mais dont le culte à mystère touchait au travail des métaux. Enfin, Hermès est "mauvais" dans une société dont tout l'idéal est guerrier. Le dieu des commerçants n'est pas un combattant. Dans la Théomachie du chant XX (35 et 72), il prend certes position en faveur des Achéens, mais il préfêre ne pas affronter Léto, la divinité pro-troyenne qui se trouve en face de lui (chant XXI, 497-501). L'aristocratie guerrière de l'époque homérique n'a guère que mépris pour les commerçants, et le sentiment est partagé par les aristocrates jusqu'en pleine époque classique. Dans les Lois (XI, 917b - 920b), Platon prend, à l'égard des commerçants, des dispositions qui témoignent de toute la méfiance qu'il leur voue. Hermès est, chez les immortels, un dieu secondaire, mais on lui reconnaît une grande célérité dans ses déplace-

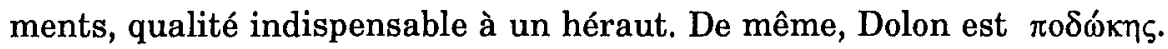

On trouve donc en présence, d'une part, Diomède et Ulysse, deux protégés d'Athéna, et, d'autre part, Dolon proche d'Hermès.

Dans les deux camps et, comme par un effet de miroir qui a été justement souligné par $\mathrm{F}$. Klinger dès $1940^{21}$, les guerriers se préparent à leur expédition. Leur armement est curieux et mérite qu'on s'y arrête un moment. Diomède emprunte son épée et son bouclier à Thrasymède, fils de Nestor; Ulysse prendra le carquois, l'épée et le casque du Crétois Mérion ${ }^{22}$.

Thrasymède est fils de Nestor et ce dernier, on l'a dit, a souvent un caractère chthonien. Mérion, lui, est un héros crétois très archaïque, comme l'est le casque en cuir de bœuf recouvert de dents de sanglier qu'il remet à Ulysse. Il s'agit d'une pièce importante que l'aède décrit avec précision et dans lequel les archéologues ont reconnu un casque mycénien ${ }^{23}$. Armement remarquable donc, mais surtout armement emprunté. En quelque sorte,

21 Über die Dolonie, in Hermes, 75 (1940), p. 337-368.

22 L'étymologie du nom Mérion reste peu claire : P. Chantraine, D.E., p. 697, s.v.

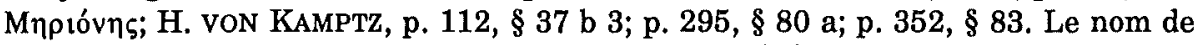

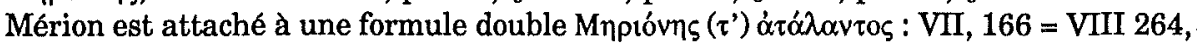
XVII 259 (II, 651). La formule est certainement ancienne, puisqu'il faut la scander

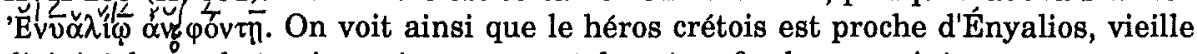
divinité du combat qui sera à un moment donné confondue avec Arès.

23 Les armes prêtées, arc et carquois, sont celles des kouroi : A. ScHNAPP-GourBeillon, Lions, héros, marques, p. 108; F.H. STUBBINGs, Arms and Armour, in A.J.B. WACE F.H. StubBInGs, A Companion to Homer, p. 513-517, pl. 32c, et BuRCHHARDT, Helme, p. 62-63, in Archaeologia Homerica, E. - Sur le caractère couréotique de la coiffure prêtée par Thrasymède, cf. L. GeRnET, Dolon le loup, in AIPhO, 4 (1936), p. 189-208 (Mélanges Franz Cumont). 
Diomède et Ulysse se déguisent. Le déguisement permet de ne pas être reconnu et d'échapper à des forces hostiles dans des moments spécialement difficiles. C'est pourquoi, d'une façon générale, les déguisements sont fréquents dans les rituels initiatiques, les rites de passage ${ }^{24}$.

C'est la même coloration que nous allons retrouver du côté troyen. Dolon revêt la peau d'un loup gris (334). Le loup est un animal sauvage, mais qui vit en société, comme l'a bien souligné Pierre Vidal-Naquet dans le Chasseur noir ${ }^{25}$. Proche d'Apollon, dieu qui patronne les jeunes en instance d'initiation, le loup est de même associé à ces cérémonies. On le retrouvera dans le nom du roi d'Arcadie, Lykaon ${ }^{26}$, à qui on attribuait la fondation des rites effrayants du Mont Lycée, rites qui avaient un caractère initiatique 27 . Le loup est gris, sans doute pour que Dolon ne soit pas vu dans la nuit. Sur sa tête, il a placé la dépouille d'une martre ( $\kappa \tau \imath \delta \varepsilon ́ \eta)$. Comme tous les mustélidés, la martre est connue pour espionner.

Autre trait qui rapproche Dolon d'Apollon : le Troyen est un archer. À un stade très ancien du mythe, les héros solitaires sont souvent des archers, sans rien perdre de leur gloire (Héraklès, Philoctète, Ulysse, etc.). En revanche, dans les combats autour de Troie, les archers ont mauvaise presse. Le tir à l'arc est le fait des êtres trop jeunes ou trop lâches ${ }^{28}$ qui ne peuvent ou n'osent aborder leurs adversaires au corps à corps. L'arc est l'arme par excellence d'Apollon, dieu des adolescents ${ }^{29}$.

Toute la scène se passe dans l'obscurité impressionnante de la nuit, autre trait qui évoque des rituels de passage.

Dolon, le rusé chasseur, croise Ulysse et Diomède «étourdiment» $(\dot{\alpha} \varphi \rho \alpha \delta i n q \sigma v, 350)$. Ceux-ci le poursuivent tels deux chiens. Comme le note

24 M. EliAde, Naissances mystiques. Essai sur quelques types d'initiation, Paris, Gallimard, 4e éd., 1959, p. 180-182. A. Schnapp-Gourbeillon, Lions, héros, masques, p. 108.

25 P. VIDAL-NAQUET, Le Chasseur noir, Paris, Maspero, 1981, p. 160, rapproche Dolon, le «loup rusén, et la légende étiologique des Apatouries. H. JEAnMaIRe, Couroi et Courètes. Essai sur l'éducation spartiate et sur les rites d'adolescence dans l'antiquité hellénique, Lille, Bibliothèque universitaire, 1939, p. 400, signale qu'Ulysse avait accompli sa première chasse au sanglier chez son grand-père maternel Autolykos dont le nom évoque le loup. Sur Dolon, le loup égorgeur et égorgé, cf. M. DETIENNE J.-P. VeRnant, La cuisine du sacrifice en pays grec, Paris, Gallimard, 1979, p. 230231.

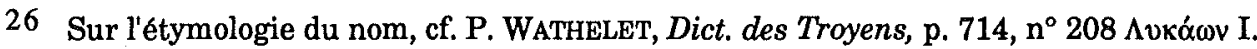

27 P. WATHELET, Homere, Lykaon et le rituel du Mont Lycée, in Les rites d'initiation. Actes du Colloque de Liege et Louvain-la-Neuve, 20-21 novembre 1984, Louvain-laNeuve, 1986 (Homo religiosus, 13), p. 285-297.

28 L'arc est aussi l'arme du lâche Pâris-Alexandre.

29 W. BURKERT, Greek Religion, p. 143-149. 
heureusement F. Lissarrague, "le chasseur est devenu gibier» ${ }^{30}$. Il y a un élément de comique possible entre la jactance passée de Dolon et son effroi : des auteurs de comédies ${ }^{31}$ et des caricaturistes ${ }^{32}$ en tireront ultérieurement un grand profit. Diomède et Ulysse, qui se cachaient parmi les morts (ćv

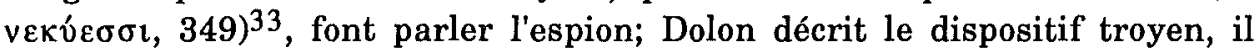
signale la présence de Rhésos, le tout dans l'espoir d'avoir la vie sauve. Il promet une immense rançon (378-381). Il y a chez lui «bronze et or et fer bien travaillé" (379), qui confirme l'impression de richesse signalée plus haut. Malgré les promesses faites, Diomède tue Dolon ${ }^{34}$. Ulysse recueille les dépouilles de l'espion troyen et les offre à Athéna.

En somme, Diomède et Ulysse, protégés d'Athéna, ont tué Dolon, très proche d'Hermès, et ceci dans l'obscurité de la nuit et avec des traits caractéristiques qui rappellent les rites d'initiation, de passage.

L'affaire n'en reste pas là. Ulysse attache les dépouilles de Dolon à un tamaris $(\mu \nu p i ́ \kappa \eta)^{35}$ qui servira de point de repère et que les Achéens retrouveront quand ils reviendront, à travers la nuit noire (468). Alors qu'on est à la dixième année du siège, Diomède et Ulysse ont eu largement le temps de se

30 Iconographie de Dolon le loup, in RA (1980), p. 3-30.

31 Cf. Euboulos, fr. 30-32 Kock, (C.A.F., II, p. 175).

32 Mentionnons un cratère en calice, italiote, à figures rouges, des environs de 380 , et conservé au British Museum (F 157) (D. WilliaMs, art. Dolon, $\mathrm{n}^{\circ} 14$ [ill.] in le LIMC). Il est attribué au Peintre de Dolon. On y voit Dolon pris entre Ulysse et Diomède. Les personnages semblent danser un ballet, ils sont séparés par des arbres dont les troncs divisent le tableau. Coiffé d'un bonnet de fourrure et vêtu d'une peau d'animal, Dolon tient un arc à la main gauche et une javeline dans la droite. Ulysse, coiffé d'un pilos, le menace, à gauche, de son glaive, tandis que Diomède, avec sur la tête un casque à panache et portant une chlamyde soulevée par le mouvement, le saisit de sa main droite; dans la gauche, il porte une lance.

33 Le détail n'est pas indifférent, il annonce ce qui va suivre. L'élément formulaire év

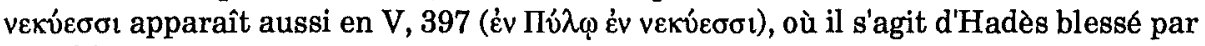
Héraklès.

34 La mort de Dolon, après sa supplication, est parallèle à celle de Lykaon, immolé par Achille. Sur son côté sacrificiel, cf. L. GERNET, Dolon le loup, p. 201; L. GERNET A. BOULANGER, Le génie grec dans la religion, Paris, 1932, p. 84.

35 A. SchNapP-Gourbeillon, Lions, héros, masques, p. 115, souligne la valeur religieuse de l'arbre, mais ne fournit aucun garant à l'appui. C'est sur la branche d'un tamaris que les chevaux d'Adraste se sont jetés, faisant tomber leur maître aux mains de Ménélas. Cf. A. MoREAU, Etymologie et mythe originel, p. 110. D'après Nicandre (Theriaka, 612), le tamaris est consacré à Apollon et à sa vertu prophétique. A.S.F. Gow et A.F. Scholfield, Nicander. The Poems and poetical Fragments, Cambridge, University Press, 1953, précisent que, d'après les scholies, les Scythes et les Mages, notamment, utilisaient le tamaris pour la divination. 
familiariser avec la plaine troyenne. Pourquoi donc placer des points de repère ? Ces repères ne sont-ils pas destinés à baliser un autre parcours plus dangereux qui justifierait tout à la fois la protection assidue d'Athéna et le caractère extraordinaire des armes revêtues par les deux héros achéens ?

Diomède et Ulysse se pressent vers le camp des Thraces. En terminant sa description des Troyens et de leurs alliés, Dolon avait mentionné "les Thraces qui viennent d'arriver à l'extrémité des lignes» (434). Ils sont commandés par leur roi, Rhésos ${ }^{36}$, fils d'Eioneus. Rhésos a les plus beaux coursiers, les plus grands qu'on ait jamais vus. Ces chevaux sont plus blancs que la neige et, pour la course, égaux aux vents. Son char est orné d'or et d'argent. Rhésos est venu porteur d'armes d'or, gigantesques, une merveille

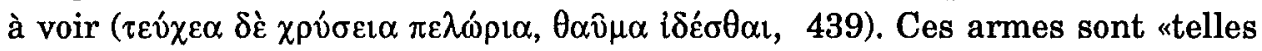
que le port en convient, non à de simples mortels, mais à des dieux immortels».

C'est en direction des Thraces uniquement que vont se diriger nos deux compères. L'anthroponyme Rhésos n'a pas de sens en grec. Depuis longtemps, on considère qu'il s'agit d'un nom thrace, d'un nom commun qui

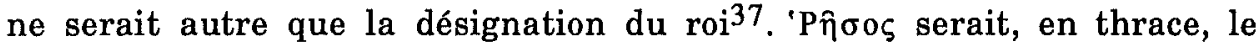
correspondant du rex, regis du latin et du rajan du sanskrit, avec passage de la palatale à la sifflante, conformément au traitement connu pour les langues satem $^{38}$. Rhésos porterait donc, comme anthroponyme, ce qui serait, dans sa langue, le nom commun du roi. On aurait là une indication que les Grecs de l'époque homérique connaissaient assez les Thraces pour leur emprunter un mot, mais pas suffisamment pour faire la distinction entre un nom propre et un nom commun. Cette impression est confirmée par le nom du

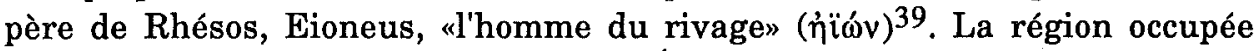
par les Thraces s'étendait au nord de l'Égée jusque dans l'actuelle Bulgarie; le fait est prouvé par la toponymie. Le nom même donné au père de Rhésos implique que les Grecs de l'époque homérique considèrent que les Thraces

36 Sur le personnage de Rhésos dans son ensemble, cf. P. WAThelet, Dict. des Troyens..., p. 943-954, n 300 'Pîoos.

37 H. vON KAMPTZ, p. 344-345, $\$ 82$ (avec bibliographie). Il existe en Troade un fleuve appelé Rhésos, mentionné par Homère (Il., XII, 20) et dans la Théogonie d'Hésiode (340). Depuis l'Antiquité, on hésite sur la situation de ce fleuve. H. von KAMPTZ, p. 344-345, $\$ 82$, ecarte l'idée que l'anthroponyme pourrait être tiré de l'hydronyme.

38 R. KATICIC, Ancient Languages of the Balkans, 2 vol., La Haye, Mouton, 1976, I, p. 143.

39 P. WATHELET, Dict. des Troyens ..., p. 563, n 138 'Hïovev́s. - La tradition ultérieure, mieux au fait de la géographie thrace, donnera le Strymon comme père à Rhésos. CONON (26 F 1 [IV] F. Jacoby) signale que le Strymon était appelé jadis Eioneus, ce qui unifie les variantes. 
occupent le rivage de la Mer Égée, ils n'en savent guère plus à leur sujet. On en vient à la même conclusion que pour Rhésos.

Le roi des Thraces est venu avec des armes d'or, gigantesques, ses chevaux et son char sont scintillants 40 . La grande taille est souvent le fait des dieux qui, sous ce rapport, dépassent naturellement et de beaucoup les mortels. Je reviendrai dans un moment sur la brillance qui entoure Rhésos, ses armes et son attelage.

Au moment où Diomède et Ulysse atteignent le camp des Thraces, ceux-ci dorment profondément (471), leurs armes bien rangées. Sans doute sont-ils épuisés par le voyage, ce qui serait normal; ce qui l'est moins, c'est que l'épopée souligne le trait et s'y attarde ${ }^{41}$. Ce profond sommeil est à l'image de la mort. Dès l'Iliade et dans toute la tradition post-homérique, on multipliera les renforts venus à l'aide des Troyens et qui tous connaissent un destin funeste; tels sont les renforts venus d'Askanie (chant XIII, 790-794) dans l'Iliade et, après cette dernière, l'arrivée de $\mathrm{Memnon}^{42}$, d'Eurypylos ${ }^{43}$ ou de Penthésilée et de ses Amazones ${ }^{44}$. Tous participent au combat avant de se faire massacrer. Ce qui distingue Rhésos et les siens de tous les autres, c'est qu'ils ne combattent pas; au contraire, ils sont tués dans leur sommeil, durant la première nuit qu'ils passent à Troie. La chose est tellement frappante que des poètes postérieurs s'en sont inquiétés. Pindare (fr. 262, B. Snell) suppose que Rhésos a combattu les Achéens au moins une journée avant de se faire tuer. La supposition du grand poète thébain est $a$ priori vraisemblable, mais, comme on va le voir, il a, pour une fois, rationalisé un mythe plus complexe.

De manière systématique, Diomède massacre des Thraces, tandis qu'Ulysse range les cadavres afin de permettre aux chevaux de se dégager. Le fils de Tydée tue douze Thraces et le treizième est Rhésos. Les nombres ne sont pas dus au hasard. Sur le plan mythique, douze désigne souvent une totalitét ${ }^{45}$.

Athéna, qui a soutenu toute l'entreprise de ses protégés, intervient spécialement en ce qui concerne Rhésos. Elle lui a envoyé un songe, en fait

40 D'autres héros possèdent un char scintillant, comme Agamemnon (IV, 226) ou Énée (V, 239), mais l'aède n'y insiste pas autrement.

41 On s'étonne aussi que, comme dans toutes les armées du monde, il n'y ait pas de gardiens qui veillent sur le sommeil des troupes. Au chant XXIV, le poème mentionne des gardes à l'entrée du camp achéen.

42 Éthiopide, 185-187, résumé de Proklos (A. Severyns).

43 Petite Iliade, 219-220, résumé de Proklos (A. Severyns).

44 Éthiopide, 175-177, résumé de PRokLOS (A. Severyns).

45 G. GermaIN, Homère et la mystique des nombres, p. 17-18. 
un cauchemar, qui occupe toute son attention ${ }^{46}$. Le malheureux se voit tué en rêve par Diomède, ce qui se passe en même temps dans la réalité. On se trouve ici en présence d'une des ruses qui caractérisent la déesse. Se faisant rassurante (elle semble avertir Rhésos par un songe), elle provoque en réalité la mort du héros. Elle agira de même au chant XXII (223-305), quand elle revêtira la figure de Déiphobe, soi-disant pour encourager Hector, mais, en fait, pour l'amener à la mort ${ }^{47}$. On peut dire que, pour la déesse, chez Homère en tout cas, la fin justifie les moyens.

Rhésos donc est mort sans avoir combattu, Ulysse s'empare de ses chevaux; quant à Diomède, il hésite. "Quelle bravade suprême pourrait-il bien oser? Se saisir du char où reposent les armes étincelantes et le tirer par le timon ou l'enlever à bras tendus ? Ou arracher la vie à d'autres Thraces encore (504-506) ?» Détail curieux, contrairement à ce que l'usage des temps aurait fait attendre, Rhésos n'a pas de cocher ${ }^{48}$.

Athéna surveille toujours la situation. Conformément à son caractère volontiers interventionniste, elle parle à Diomède, sans prendre de déguisement, pour lui conseiller de faire retraite immédiatement. Les deux Achéens s'empressent d'obéir, ils sautent sur les chevaux et fuient.

Il était grand temps. Comme le dit l'aède, «ce n'était pas en aveugle que veillait Apollon ${ }^{49}$, le dieu à l'arc d'argent» (515); il éveille Hippokoon, un

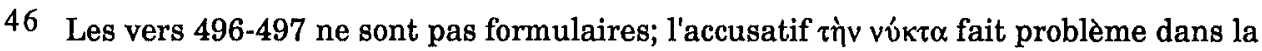
mesure où il s'agit de désigner, non la nuit entière, mais un moment de la nuit. W. LEAF, The Iliad, ed. with English Notes and Introduction, Londres, Macmillan, 2e éd., 1900, I, p. 459, qui condamne le vers, note, de plus, qu'Homère respecte en général la vraisemblance en faisant apparaître au dormeur seulement des personnages qui lui sont connus, ce qui ne serait pas le cas ici. Même opinion chez K.F. AMEIS - C. HenTZE, Homers Ilias, I, 4, 5e éd., Leipzig, Teubner, p. 36. On notera,

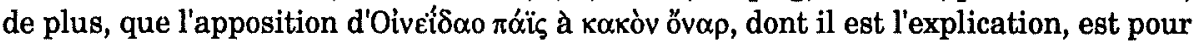
le moins maladroite. Tout se passe comme si, ici comme ailleurs, l'aède qui quitte le style formulaire était empêché de trouver une expression claire et complète. Il est possible aussi qu'en utilisant l'accusatif vúkr $\alpha$ l'aède ait voulu insister sur la longueur du rêve qui aurait tenu Rhésos toute la nuit.

47 Elle avait de même pris l'apparence de Laodokos pour tromper Pandaros (IV, 86-88). Ce genre de tromperie de la part des dieux apparait aussi dans le domaine de l'Inde, spécialement dans le mythe de Yayäti : M. DEFOURNY, Le mythe de Yayäti dans la littérature épique et purānique. Étude de mythologie hindoue, Paris, 1978 (Bibliothèque de la Fac. de Phil. et Lettres de l'Université de Liège, fasc. 221), p. 153.

48 De même, Priam ira trouver Achille en char et sans cocher, mais il s'agit d'une histoire tout à fait extraordinaire (cf. P. WATHELET, Priam aux Enfers).

49 On pourrait croire qu'ici comme en d'autres passages de l'Iliade Apollon est malavisé : c'est lui qui, au chant XX, opposera Énée à Achille, alors que le destin du fils d'Anchise n'est pas de mourir. Il faudra toute la pondération de Poséidon, pourtant allié des Achéens, pour sauver Énée et respecter les destins. 
cousin de Rhésos, qui donne l'alarme. Cet Hippokoon a manifestement été

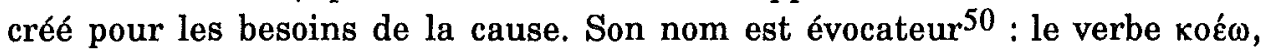
dont provient le dérivé "percevoir», spécialement dans le domaine religieux. La même racine donne en sanskrit le mot kaví, qui désigne un poète, un inspiré. En grec, plusieurs termes issus de ce radical sont liés à des fonctions sacrées, il s'agit

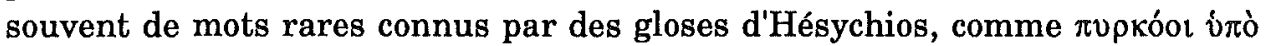

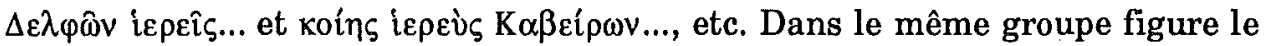
nom du prêtre troyen connu par la tradition posthomérique, le célèbre $\Lambda \alpha 0$ кó $\omega v$. Cette connotation religieuse pourrait également se retrouver dans certains emplois archaïques de cavere ou de ses dérivés en latin. Bref, Hippokoon serait "celui qui a une perception sacrée, religieuse des chevaux". Ce qui convient effectivement à la situation dans laquelle il apparaît.

Le vers où il est dit qu'Apollon ne veillait pas en aveugle est curieux. On se fait la réflexion que le dieu à l'arc d'argent n'est pas particulièrement rapide dans ses réactions. À première vue, il intervient à la manière des «Carabiniers d'Offenbach», puisque Rhésos est déjà tué, à moins qu'il n'y ait à sauver quelque chose de plus important que Rhésos : par exemple son char, dont Diomède caresse un moment le projet de s'emparer.

On se souviendra que, lorsque Dolon s'est offert pour aller espionner les Achéens, il a fait à Hector une demande extravagante : le char et les chevaux d'Achille. Comme l'espion l'avoue à Ulysse et à Diomède au moment où il est arrêté, Ulysse se moque de ses prétentions. D'un côté, Dolon voulait s'emparer du char d'Achille et de ses chevaux immortels; de l'autre, Diomède et Ulysse prennent les chevaux de Rhésos et Diomède est tenté de s'emparer de son char. J'ai souligné au début que la Dolonie comportait une action en miroir.

Alors qu'Hippokoon donne l'alarme et que les Thraces constatent les dégâts, les deux héros achéens se sont enfuis ${ }^{51}$. Ils suivent au retour le chemin qu'ils avaient pris à l'aller et récupèrent les dépouilles de Dolon qu'ils avaient laissées comme point de repère.

50 P. WATHELET, Dict. des Troyens, p. 614-617, nº 172 'I $\pi \pi$ okówv.

51 Diomède et Ulysse fuient en chevauchant les coursiers de Rhésos. Après ARISTONIKos (PTQ en $\varepsilon$ 371), EUSTATHe (Ad Od. $\varepsilon 371,1539,31$ ) remarque qu'Homère connaît lui-même les chevaux de course, mais qu'il ne décrit pas de héros qui s'en servent, si ce n'est dans un cas d'absolue nécessité, comme Diomède dans la Dolonie. Cf. A. Severyns, Le Cycle épique dans l'école d'Aristarque, Liège, VaillantCarmanne, 1928, p. 128. - À la limite, "i $\pi \pi 01$ désigne aussi un char, mais le contexte implique que Diomède et Ulysse chevauchent. Cf. A. ScHNAPP-GouRBEILLON, Lions, héros, masques, p. 117. 
C'est le vieux Nestor qui est le premier à les accueillir, Nestor dont le personnage, on l'a dit, est lié à Pylos, la porte de l'Au-delà. Nestor s'étonne devant la beauté des chevaux pris à Rhésos : «Un dieu serait-il venu vous les offrir ? Ils rappellent terriblement les rayons même du soleil», $\alpha i v \hat{\omega} \varsigma$

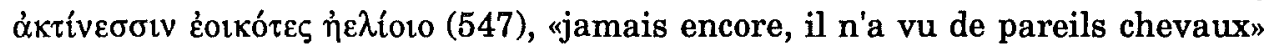
(550).

Diomède, Ulysse et leurs prises passent le fossé (564) - le fossé a souvent une valeur mythique, comme un cours d'eau ${ }^{52}$, on installe les chevaux de Rhésos dans l'écurie de Diomède, puis les deux héros prennent deux bains, un dans la mer et l'autre dans des baignoires. Le trait prend une allure de rite de purification ${ }^{53}$. Ensuite, ils s'assoient pour dîner, non sans avoir fait une libation à Athéna. Ces derniers traits soulignent le côté religieux de la scène. Nous n'aurons pas le mauvais goût de noter que c'est le troisième dîner d'Ulysse cette nuit-là. Il avait déjà eu deux repas du soir au chant IX, l'un chez Agamemnon et l'autre chez Achille!

Vue à travers notre logique, la Dolonie est pleine d'incohérences, mais notre logique n'est pas celle d'Homère, ni celle du mythe. L'aède insiste sur la brillance de l'attelage de Rhésos : ses armes, ses chevaux, son char sont éclatants de blancheur, ruisselants d'or et d'argent, plus blancs que la neige, semblables aux rayons du soleil. On dirait qu'il s'agit de l'équipage d'un dieu. Cet éclai est d'autant plus remarquable que tout l'épisode se passe de nuit, dans l'obscurité.

La question vient à l'esprit : ce char si resplendissant n'est-il pas le char du Soleil, char que le dieu conduit lui-même sans cocher ?

La mythologie du XIXe siècle et du début du XXe siècle a usé et abusé des mythes solaires; il convient donc de se montrer très prudent, mais on ne peut, passant d'un excès dans l'autre, nier complètement l'existence de tels mythes. Les Grecs ont connu un culte solaire, notamment à Rhodes ${ }^{54}$. Hélios est un dieu qui joue un certain rôle, notamment dans l'Odyssée; il semble

52 P. BRUNEL, L'évocation des morts et la descente aux Enfers. Homère, Virgile, Dante, Claudel, Paris, Sedes, 1974, p. 86-87. Sur le symbolisme des limites, M. EliadE, Traité d'histoire des religions, Paris, Payot, 1953, p. 318-319.

53 Sur le sens de la purification double d'Ulysse et de Diomède après leur expédition, cf. J.-P. VERNANT, Le pur et l'impur, in L'Année sociologique (1953-54), p. 331-352 (= Mythe et société en Grèce ancienne, p. 121-140, spécialement p. 122). - A. ScHNAPPGOURBEILLON, Lions, héros, masques, p. 118, signale que les héros se purifient dans la mer parce que la souillure est grave, ce qui mettrait encore en valeur l'importance de Rhésos.

54 L.R. FARNELL, The Cults of the Greek States, Oxford, Clarendon, V (1909), p. 418. 
que le Soleil ait été honoré à l'époque minoenne ${ }^{55}$. En outre, il existait apparemment un culte du Soleil en Thrace ${ }^{56}$.

Plusieurs conceptions existaient quant à ce que faisait le soleil durant la nuit. Parfois, comme on le trouve sur des peintures de vases, il va d'ouest en est dans une nacelle qui vogue sur le fleuve Océan, parfois aussi le soleil passe la nuit, «dort» si l'on ose dire, dans l'Autre Monde, d'où il ressortira le matin. Une telle opinion semble impliquée par un passage de l'Odyssée. Au chant X (190), Ulysse déclare : "Amis à cet endroit, nous ne pouvons rien voir, ni le point de noroît, ni celui de l'aurore. Où tombe sous la terre le soleil des vivants ? Par où nous revient-il ?» L'idée est reprise par Apollonios de Rhodes (III, 1191) : "le soleil s'enfonçait au loin sous la terre obscure, pardelà les dernières cimes des Éthiopiens occidentaux» ${ }^{57}$.

Au moment où il est tué par Diomède, Rhésos, le roi venu de Thrace, pays qui possède probablement un culte solaire, est prolongé dans un sommeil très profond. Des sources antiques postérieures à Homère rapportent que, si Rhésos avait combattu un seul jour à côté des Troyens, ils auraient été invincibles $^{58}$. Il fallait donc que Rhésos pérît dès son arrivée ${ }^{59}$. Le Soleil est, pour la tradition épique, un symbole de permanence. Il est le dieu qui, dans sa course diurne, voit tout. Dans l'Odyssée (chant VIII), Arès et Aphrodite l'apprennent à leurs dépens, puisque c'est le Soleil qui annonce son infortune à Héphaïstos et qui l'avertit que les amants sont réunis. Il est surtout qualifié d'«infatigable" 'Hé $\lambda$ เov $\delta$ ' $\left(\tau^{\prime}\right) \dot{\alpha} \kappa \alpha ́ \mu \alpha v \tau \alpha$. La formule est attestée, non seulement dans l'Iliade (XVIII, 239 et 484), mais aussi chez Hésiode (Théogonie, 956), ce qui montre qu'il s'agit d'un trait bien connu.

Plusieurs héros grecs, à un stade très archaïque de la mythologie, tentent de s'emparer du char solaire. Si la légende de Phaéthon est célèbre, on mentionnera, dans le même groupe et chaque fois avec des variantes,

55 La question a été beaucoup débattue (M.P. NILSSON, Geschichte der griechischen Religion, 2e éd., Munich, Beck, 1955, p. 301-302). Certains indices, comme le nom de Pasiphaé, femme de Minos, semblent aller dans ce sens (cf. JESSEN, art. Helios, in RE, VIII [1912], c. 73).

56 SopHocle, Téreus, fr. 582 (523) St. Radt.

57 RAPP, art. Helios, in W.H. ROSCHER, Ausfiuhrliches Lexikon..., I, c. 2011; JESSEN, art. Helios, in RE, VIII (1912), c. 90-93.

58 Tragédie Rhésos, 600-605; Schol. A en K 435; EustatHe, Ad Il. K 435, 817, 26; d'après VIRGILE, Enéide I, 469-473, les chevaux de Rhésos devaient être pris avant d'avoir pu boire l'eau du Xanthe; cf. aussi Servius, Ad AEn., II, 13.

59 On a signalé plus haut que, pour PINDARE, fr. $262, \mathrm{Br}$. Snell, cité par les scholies $\mathrm{b}\left(\mathrm{BCE}^{3}\right) \mathrm{T}$ en $\mathrm{K} 435$, Rhésos a combattu les Grecs pendant une journée et qu'il a été tue la nuit suivante, à la suite d'une intervention d'Héra et d'Athéna (cf. EusTATHE, Ad Il. $K 435,817,28$ ). 
Atymnios ${ }^{60}$ (fils d'Amisodaros, tué par Antiloque, mais aussi héros à Gortyne), Ténagès ${ }^{61}$ (à Rhodes), Akamas, fils d'Anténor, tué par le Crétois Mérion ${ }^{62}$. Diomède lui-même est un héros qui appellerait un long commentaire, mais on se bornera ici à deux indications. Dans la Thébaïde du Cycle épique ${ }^{63}$, Tydée, père de Diomède, est blessé par Mélanippos. Athéna, qui protégeait le père comme elle protégera le fils, tente de lui apporter l'ambroisie pour le sauver. Comme elle découvre Tydée occupé à humer la cervelle de Mélanippos, elle se détourne, mais Tydée implore la déesse afin qu'elle réserve l'immortalité au moins à son fils.

Dans l'ensemble de l'Iliade, Diomède fait un grand massacre de Troyens; beaucoup de ses victimes présentent des points communs. Tantôt, il fait sa proie d'un groupe de deux combattants comme c'est le cas pour Astynoos et Hypérion (V, 144-147) ${ }^{64}$, pour Xanthos et Thoon (V, 152-158) ${ }^{65}$ on songe aux deux personnages qui se trouvent naturellement sur un char, le combattant et son cocher ${ }^{66}$-, tantôt il est précisé que les Troyens se trouvent sur un char, comme c'est le cas pour Échemmon et Chromios ${ }^{67}$, fils de Priam (V, 159-165), pour Pandare et Énée (V, 166-330), pour Axylos et Kalésios (VI, $12-19)^{68}$, pour Agélaos (VIII, 253-260) ${ }^{69}$ ou pour Thymbraios (XI, 320-327) ${ }^{70}$ ou encore pour les fils de Mérops, Adraste et Amphios (XI, 328-334). Les victimes de Diomède sont liées à Apollon, voire au Soleil. C'est le cas pour Astynoos et Hypérion, pour Abas et Polyeidos et aussi pour Pandaros, l'archer lycien auquel Apollon a donné son arc. Il tuera encore Agélaos, dont le nom évoque l'Apollon colonisateur, Thymbraios, lié à l'Apollon de Thymbra, les deux fils du devin Mérops de Percote.

Que Diomède, qui est de surcroît qualifié en plusieurs passages de i $\pi \pi \delta ́ \delta \alpha \mu \circ$, "dompteur de chevaux», tue Rhésos, proche du Soleil, et forme le

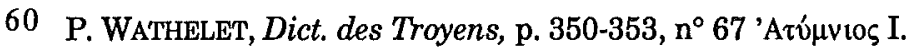

61 H. von GetsAU, art. Tenages, in Der Kleine Pauly, V, c. 584. - O. GRUPPE, Griechische Mythologie, Munich, Beck, 1906, p. 269.

62 P. WATHELET, Dict. des Troyens,, p. 238-240, n 17 'Aкó $\mu \alpha \varsigma$ I.

63 A. SEveryns, Le Cycle épique dans l'école d'Aristarque, Liège-Paris, 1928, p. 219-220.

64 P. WATHELET, Dict. des Troyens, p. 349, $\mathrm{n}^{\circ} 65$ 'Aørúvooৎ II.

65 P. WATHELET, Dict. des Troyens, p. 578-579, n’ 149 Өówv I.

66 On a souligné que, dans l'Iliade, Rhésos n'a pas de cocher. Voulant rationaliser la légende, l'auteur de la tragédie lui en donnera un, auquel il prête un certain rôle (Rhésos, 729-876); c'est lui qui annonce à Hector la mort de Rhésos.

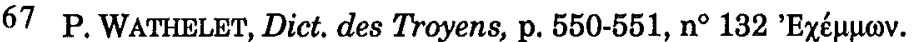

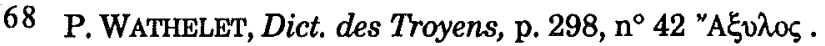

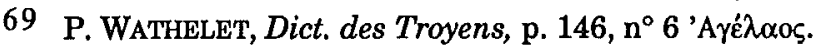

70 P. WATHELET, Dict. des Troyens, p. 584, $\mathrm{n}^{\circ} 154$ $\theta 0 \mu \beta$ poîos. 
projet de s'emparer de son char est donc dans la logique du personnage. Que le meurtre ait lieu de nuit, alors que le Soleil se trouve dans l'Autre Monde correspond sans doute à une conception du temps. Diomède est accompagné par Ulysse, autre héros qui voyage vers l'ouest, c'est-à-dire vers le pays des morts. Faut-il rappeler la Nekyia de l'Odyssée ? Les deux héros sont protégés par Athéna, la déesse qui ne cesse de les patronner tout au long de l'Iliade et de l'Odyssée; elle l'avait fait auparavant pour Tydée, le père de Diomède. Athéna est la déesse qui aide les héros dans leurs exploits et leur permet de traverser les régions obscures, comme le regard de sa chouette perce les ténèbres de la nuit. C'est aussi la déesse qui voulait conférer l'immortalité à Tydée.

En somme, Diomède et Ulysse sont descendus dans l'Autre Monde pour aller y tuer Rhésos, héros solaire. Si celui-ci avait combattu au côté des Troyens, ces derniers auraient eu avec eux l'immortalité, c'est dire que les Achéens n'auraient jamais pu les vaincre.

Que vient alors faire dans cette histoire l'épisode de Dolon ? Etait-il bien nécessaire de tuer Dolon pour arriver à Rhésos ? Après tout, Athéna ou quelque devin aurait pu attirer l'attention des Achéens sur le péril que leur faisait courir l'arrivée du roi thrace dans le camp troyen. On a eu l'occasion plus haut de souligner que Dolon était très proche d'Hermès. L'opposition entre Diomède et Ulysse, d'un côté, et Dolon, de l'autre, reflète une opposition entre Athéna et Hermès. Athéna est constamment citée dans la Dolonie, mais, d'Hermès, il n'est fait aucune mention explicite. Et pourtant, j'ai trouvé, chez les auteurs anciens, la confirmation de mon explication.

La Dolonie a suscité un certain nombre d'illustrations dans la peinture attique à figures noires et à figures rouges, de même que dans la céramique italique, où la scène devient parfois burlesque, s'inspirant sans doute de comédies comme l'a bien rappelé $F$. Lissarrague ${ }^{71}$. Le vase qui m'intéresse est une coupe à figures rouges (Paris, Cabinet des Médailles, 526, 743, 553, 641), des années 500-490. Il est signé du potier Euphronios et est attribué par Sir John Beazley à Onésimos, le Peintre de Panaitios ${ }^{72}$. Il montre le moment où Dolon, pris entre les Achéens, va être mis à mort. Or, à droite de la scène, on voit Athéna en armes qui triomphe et, de l'autre, à gauche, Hermès qui s'enfuit. Telle est du moins la reproduction du vase qui est fournie par le

71 Iconographie de Dolon le Loup, p. 27. - Sur la céramique italiote, voir J.-M. MORET, L'Ilioupersis dans la céramique italiote. Les mythes et leur expression figurée au IVe s., Institut suisse de Rome, 1975, I, p. 213.

72 D. WILLIAMS, art. Dolon, $\mathrm{n}^{\circ} 11$ (ill.), in le LIMC. 
dictionnaire de Roscher ${ }^{73}$, mais il faut toujours être prudent : l'examen d'une autre reproduction du même vase montre que la figure d'Hermès est, pour la plus grande part, l'effet d'une restauration. Le document doit néanmoins être pris en compte car il subsiste, comme authentique, l'extrémité du pied à talonnette ailée du dieu héraut.

Pourquoi faut-il tuer Dolon/Hermès pour atteindre Rhésos ? Hermès est le dieu psychopompe qui, dans l'ordre normal des choses, conduit les âmes des morts dans l'Autre Monde. L'expédition de Diomède et d'Ulysse n'est pas de ce modèle. C'est une sorte de coup de main qui ramènera les deux héros achéens, bien vivants, à leur base de départ. Ainsi s'explique que le récit du meurtre de Dolon contient un certain nombre d'allusions à des rites de passage; le passage suprême consiste bien à se rendre dans l'Autre Monde. L'importance de l'enjeu représenté par la quête des deux Achéens est soulignée par l'accumulation des détails indicatifs qu'il faut se garder de prendre pour de vains enjolivements.

Il est un point sur lequel je voudrais m'arrêter encore un instant : je viens de défendre la thèse selon laquelle le Soleil est éternel; de même, par nature, Hermès appartient au groupe des immortels; or, je ne puis nier l'évidence, Dolon et Rhésos périssent. Le travail que je viens de terminer sur les Troyens de l'Iliade ${ }^{74}$ m'a révélé un phénomène très répandu dans l'épopée grecque : beaucoup de héros sont en quelque sorte l'incarnation mortelle de divinités immortelles. Sarpédon, fils de Zeus, représente le Cronide auprès des Troyens, Hector a originellement des affinités avec Arès, Euphorbe est une figure d'Apollon. La liste serait longue de tels ensembles de divinités et de mortels. Ceci rejoint une remarque incidente, mais riche d'enseignements que fait Walter Burkert ${ }^{75}$. Il souligne que souvent, dans le rituel, un dieu est doublé par un héros qui meurt parfois sous ses coups : Hyakinthos est un doublet d'Apollon tué par lui, Iphigénie est Artémis elle-même, qui, en quelque sorte, cause sa propre mort.

La religion grecque représente un ensemble de divinités dont les domaines se recoupent et qui s'efforcent perpétuellement de rétablir entre elles un minimum d'équilibre, sans jamais cesser de s'opposer. La rivalité entre les dieux trouve dans les récits épiques une expression commode. Ce

73 voN SYBEL, art. Dolon, in W.H. RoSCHER, Ausführliches Lexikon..., I, c. 1195-1196, qui reproduit un dessin d'OVERBECK, Gall. her. Bildwerke, pl. 17, fig. 2.

74 Ce travail sera publié en deux ouvrages, actuellement sous presse : Dictionnaire des Troyens de l'Iliade, Liège, 1988 (Instrumenta et Documenta, 1) et Les Troyens de l'Iliade. Mythe et histoire, Liège, 1989 (Collection de la Faculté de Philosophie et Lettres).

75 Greek Religion, p. 188-189. 
qui semble souvent de banals combats, surtout dans l'Iliade, constitue l'écho plus profond de ces luttes entre les dieux. L'avantage est, pour ainsi dire, qu'à l'inverse de ce qui se passe pour les dieux, on peut faire mourir les héros.

Dans l'hypothèse que je viens de développer, la Dolonie dans son ensemble constituerait une sorte de morceau de bravoure, un peu comme les Catalogues du deuxième chant. C'est un morceau qui a été inséré dans l'Iliade, parce que sans doute le public l'attendait. L'insertion n'est pas plus adroite que pour les Catalogues, mais, encore une fois, gardons-nous d'appliquer à l'épopée homérique des critères de jugement qui sont nôtres.

Manifestement, la Dolonie atteste une concentration plus grande de traits mythiques que l'Iliade. Est-elle antérieure ou postérieure à Homère ? Certains traits de langue du passage ont été rapprochés de l'Odyssée et, en 1937, Pierre Chantraine ${ }^{76}$ a souligné le caractère considéré par lui comme tardif de ces faits linguistiques. Le déchiffrement du linéaire $B$ a pu remettre en cause des opinions formulées en 1937; de surcroît, je ne suis pas absolument sûr que l'Odyssée présente constamment un niveau de langue plus tardif que l'Iliade; la question est délicate à trancher. Les similitudes linguistiques entre la Dolonie et l'Odyssée pourraient également trouver leur justification dans le sujet des deux cuvres, deux descentes aux Enfers. Avant de s'avancer plus loin, il faudrait étudier soigneusement le langage traditionnel de la Dolonie et peut-être y verrait-on alors plus clair.

Je me réserve de faire plus tard ce travail; aussi, sur ce point, resterai-je sur une prudente réserve, me bornant à souligner qu'au moins au plan interne la Dolonie a sa propre cohérence, contrairement à ce qu'on a souvent affirmé.

Paul WATHELET

Université de Liège

Section de Philologie classique

Place du XX-Août, 32

B - 4000 LIEGE

76 Remarques critiques et grammaticales sur le chant $K$ de l'Iliade, in Mélanges Derrousseaux, Paris, 1937, p. 59-68. 